\title{
Collaborative Tactical Planning in Multi-level Supply Chains Supported by Multiagent Systems
}

\author{
Jorge E. Hernández ${ }^{1}$, Raúl Poler ${ }^{1}$, Josefa Mula ${ }^{1}$, and David de La Fuente ${ }^{2}$ \\ ${ }^{1}$ CIGIP (Centro de Investigación Gestión e Ingeniería de Producción), Dpto. Organización de \\ Empresas, Escuela Politécnica Superior de Alcoy, Universidad Politécnica de Valencia, \\ Edificio Ferrándiz y Carbonell, 2, 03801 Alcoy (Alicante), Spain \\ \{jeh, rpoler, fmula\} @cigip.upv.es \\ ${ }^{2}$ Dpto. de Administración de Empresas, Escuela Politécnica Superior de Ingenieros \\ Industriales, Universidad de Oviedo, Campus de Viesques s/n. 33204 Gijón (Asturias), Spain \\ david@uniovi.es
}

\begin{abstract}
In the supply chain modeling context, the agent-based model aims to represent not only each node, but also the information sharing process among these nodes. Despite the complexity of the configuration, the agent-based model can be applied straightforwardly to support the collaborative planning process. This allows the parties to achieve common goals effectively. Thus by sharing accurate, action-based information, collaboration among the nodes will emerge to improve the decision-making process in supply chain planning processes. Therefore, this paper presents a novel collaborative planning model in multilevel supply chains that considers a multiagent system modeling approach to carry out the iterative negotiation processes which will support the decisionmaking process from a decentralized perspective.
\end{abstract}

Keywords: Collaborative planning, supply chain management, negotiation, multiagent systems.

\section{Introduction}

The decisions linked to the supply chain management process can affect three decisional levels: strategic, tactical and operational. The first relates with long-term decisions, which include design processes. The second implies decisions at the mid-term level, which will influence planning activities at the operational level. Finally, the third mainly involves the execution of planned activities, where a decision has to be made from a short-term perspective. Thus, high uncertainty in the planning horizon will have a significant effect at this level because, in most cases, there is not enough reaction time to meet the new requirement. Thus, the system will need to consider high inventory levels to cover this uncertainty, which will imply and increase cost rates. This impact may be stronger if the system is composed of a multi-level supply chain where suppliers, warehouses, 3PL, retailers, etc., are directly affected by strategic and tactical decisions. Hence, a system should be studied from three viewpoints in relation to its products, information and decision flows [6]. Therefore, the on-time 
and right decisions in the tactical planning process might avoid, and also optimize, these unnecessary levels and rates. Furthermore, [12] established that tactical planning covers those planning decisions in which short-term decisions are defined, such as synchronized planning policies for procurement, production, distribution and sales. In a supply chain context, it is possible to state that the planning process takes into account the coordination and integration of the key business activities which are normally undertaken by enterprises, ranging from the procurement of raw materials to the distribution of final products to end customers [5]. In addition, lead times are one of the important factors to be considered in the supply chain planning process, as suggested by [1]. Thus, it is important to consider lead times at the tactical level because lead times vary in terms of environmental uncertainties, which is precisely one of the serious factors that challenge suppliers and buyers in a supply chain when it comes to defining the mean and variance. In this context, supply chain management offers new opportunities for conducting operations strategy research [17]. Nevertheless, supply chain management at the information system level is becoming a high core competency, and enterprise resource planning systems are expected to become an integral component of supply chain management. Moreover, installing such systems is expensive and risky, and managers must decide how to use their limited resources and how to invest in the right products [15].

Therefore, this paper is set out as follows: Section 2 briefly reviews the relevant literature on collaborative planning and multi-agent systems in a supply chain management context. Section 3 establishes the formal formulation for the novel collaborative planning proposal in multi-level supply chains. Section 4 provides a brief example of the proposal application. Finally, Section 5 offers the main conclusions drawn and also briefly describes our future work.

\section{Background}

Despite the complexity of these systems, there are significant benefits at the collaborative level from a supply chain perspective, where the information exchanging process occupies a relevant place in supporting the planning processes among the nodes. Hence, as defined by [6], collaborative activities also imply a distributed decision-making process which involves several supply chain nodes. So in relation to the collaboration that may exist among these supply chain nodes, planning will consider the answers that customers send to the manufacturer which, in turn, will be sent to suppliers; thus, messages will flow effectively. The planning process is then supported by considering more effective answers in relation to demand/orders, and the agreement processes may also be supported by considering the corresponding negotiation processes [7].

In addition, this collaborative process can be seen from two perspectives: centralized and decentralized. From the centralized perspective, a virtual node controls the supply chain information process to support the planning processes. An example of this is highlighted in [11]. Then, from the centralized viewpoint, however, collaborative planning (among supply chain partners) can be achieved by simple coordinated upstream planning by providing the collaboration partners an opportunity to modify the suggested order/supply patterns iteratively [2]. Meanwhile from a decentralized 
collaboration viewpoint, each node will consider its collaborative and non collaborative partners (customers and suppliers) to carry out its planning processes [13]. This last perspective leads to a more realistic current supply chain process. In addition, examples of this decentralized perspective relating to logistic supply chain planning, supply chain coordination, a collaborative supply chain decision-making model and a supply chain coordination under demand uncertainty, can be found in [9], [3], [8] and [16], respectively.

Consequently, and as mentioned earlier, information technology systems must be set up irrespectively of the collaborative approach that the supply chain possibly considers to support such aspects as the information flow among the nodes in order to support supply chain planning and the communication processes. In the same context, and by considering the robustness presented by the multi-agent system, [10] proposed a novel architecture to support the inter-enterprise functions/resources integration and collaboration in a networked context. Moreover, [4] present auctions experiments, supported by multiagent systems, to view the impact at the operative level. To support this, modeling methodologies like MASCOT [14] or SCAMM-CPA [7] can be considered where a full literature review has been contemplated. Thus, the aim of multiagent proposals is to support the development of a system with a collaborative purpose by exchanging information without sharing a common system, but one that also considers the related ontology's with a view to supporting the communication process, hence leading to collaborative behavior. Therefore, this technology has been considered to support the following proposal.

\section{The Collaborative Tactical Planning Formulation in Multi-level Supply Chains}

The proposed planning process is about to consider the right and on-time information to support the decision-making process. Thus, the decision-making process is supported by a manufacturing requirement planning (MRP) system which, like the main information for all the $\mathrm{n}$ periods, considers inputs such as demand (D), inventory level (IL), the sell price (SP) and capacity (CAP). On the other hand, the main MRP outputs are the following: planned orders (PO), Delay on Demand (DoD), Profit and SP. Then, each node considers its own MRP mechanism (M).

The collaborative perspective of this proposal, as seen in Fig. 1, establishes communication at the multi-level area of the supply chain by considering the customer node's PO, which will be contemplated as D in the directly relating tier suppliers, as will the PO relating with the CAP information that the customer owns.

So the main idea is support a negotiation process by incrementing the SP with an increased $\mathrm{P}$ factor. In fact, if no agreement is reached among the nodes, the proposal will be improved by raising the SP.

Hence from the decentralized and collaborative perspectives, each node in the multi-level decision-making process considers its own database (or information repository) to link the communication process. This kind of communication is considered decentralized and collaborative because. 


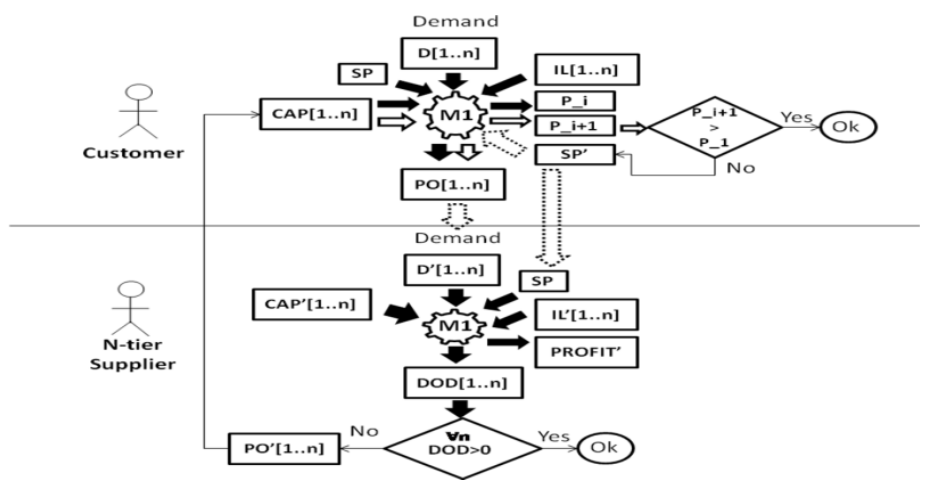

Fig. 1. Collaborative planning mechanism

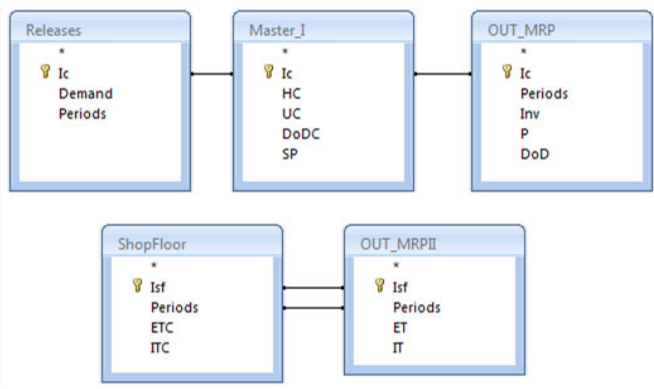

Fig. 2. Structure of the database supporting decentralized collaborative planning

In addition, Fig. 2 shows how the information system structure is composed of five main tables. These tables aim to collect the inputs and outputs generated by the MRP mechanism. In this case, the Releases table is about demand information. The Master_I table includes the information related to holding costs (HC), unitary costs UC, delay on demand costs (DoDC) and the sell price (SP). The OUT_MRP table collects information relating to the final level of the IL, P and DoD. This table holds information that will support the decision-making process of the planning process.

MRP systems also consider that information relating to ShopFloor activities, which mainly relate to CAP, ETC and ITC. Then, each answer the system generates will be restricted by the information in this table. Thus given the collaborative planning proposal, this information will consider the answer from the tier supplier to evaluate the planning proposal's level of profit. Then along with the OUT_MRPII table, the supply chain will be able to evaluate its decision by considering the right and on-time information in an iterative decentralized negotiation process.

$$
i c=\sum_{N=1}^{M} \sum_{i=1}^{n}\left(O U T_{-} M R P . I n v_{N i} * \text { Master_I. } H C_{N i}\right)
$$




$$
\begin{aligned}
& p c=\sum_{N=1}^{M} \sum_{i=1}^{n}\left(O U T_{-} M R P . P_{N i} * \text { Master_I.UC } C_{N i}\right) \\
& d c=\sum_{i=1}^{n}\left(O U T \_M R P . D o D_{1 i} * \text { Master_I.DoDC } C_{N i}\right) \\
& \text { extratc }=\sum_{i=1}^{n}\left(\text { OUT_MRPII.ET } T_{N i} * \text { ShoopFloor. ETC } C_{1 i}\right) \\
& \text { idletc }=\sum_{N=1}^{M} \sum_{i=1}^{n}\left(\text { OUT_MRP.IT } T_{N i} * \text { ShoopFloor. ITC } C_{N i}\right) \\
& s r 1=\sum_{i=1}^{n}\left(\text { Releases. Demand } 1 i * \text { Master_I.SP } P_{1 i}\right) \\
& s r 2=\sum_{i=1}^{n}\left(M R P . D_{0} D_{1 i} * \text { Master_I.SP} P_{1 i}\right)
\end{aligned}
$$

Finally, the profit level (8) will lead not only to the negotiation process, but also to the decisions relating to the planning process in each $\mathrm{n}$ period of the planning horizon. The calculus of this profit consider the following information for each period: inventory costs at each period (1), production costs (2), delay on demands costs (3), extra time costs (4), costs of the idle time of resources (5) and sell revenues (6 and 7). Thus by considering the answers from suppliers, the customer node will evaluate renegotiation by taking the new profit value which is directly affected by the raised product's sell price. The negotiation process will finish when new price changes no longer have an impact on the profit level or when no DoDc for the suppliers exist. Hence, an example of the application of this novel proposal to a multi-level supply chain is briefly provided in the following section.

\section{A Multiagent-Based System to Support Collaborative Tactical Planning in Multi-level Supply Chains}

The collaborative planning model (Fig. 3) for supply chain networks which considers a multiagent system modeling approach is supported by MPL/CPLEX, JADE 3.6 and ECLIPSE. The communication process among agents is supported by behaviors that aim to generate demands and firm orders, and to also send and receive the corresponding messages, by considering the FIPA standard communication protocols, which are mainly supported by the Contract-Net protocol. The system has been tested in a real concurrent environment, thus each agent considers its own thread.

As Fig. 3 illustrates, each node considers its own planning mechanism, as well as its own database (DB) repository. In addition, each agent is independently governed 
by its own behaviors, including its wishes and beliefs. Next, the multi-level supply chain is composed of the customer agent (CA), the first-tier supplier agent (FTSA1) and two second-tier supplier's agents (STSA1 and STSA2). Then, each node will validate its proposals by obtaining the related values at the profit level.

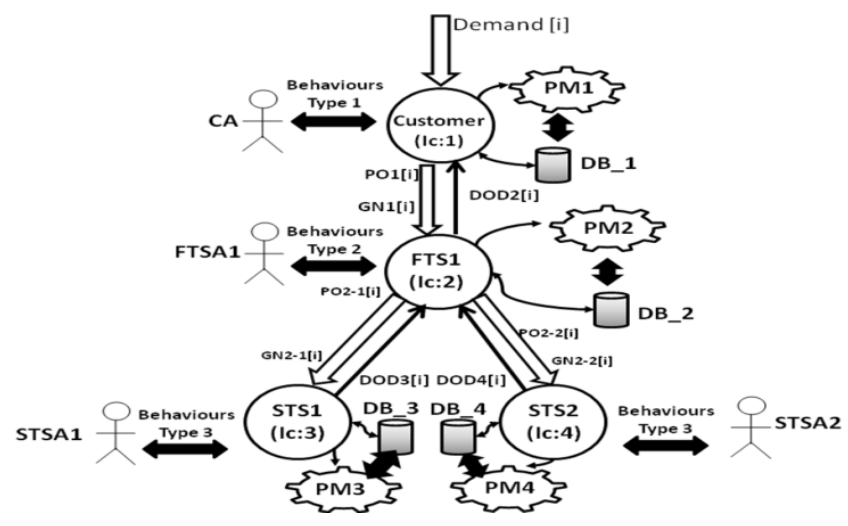

Fig. 3. A Multi-Tier Supply Chain example

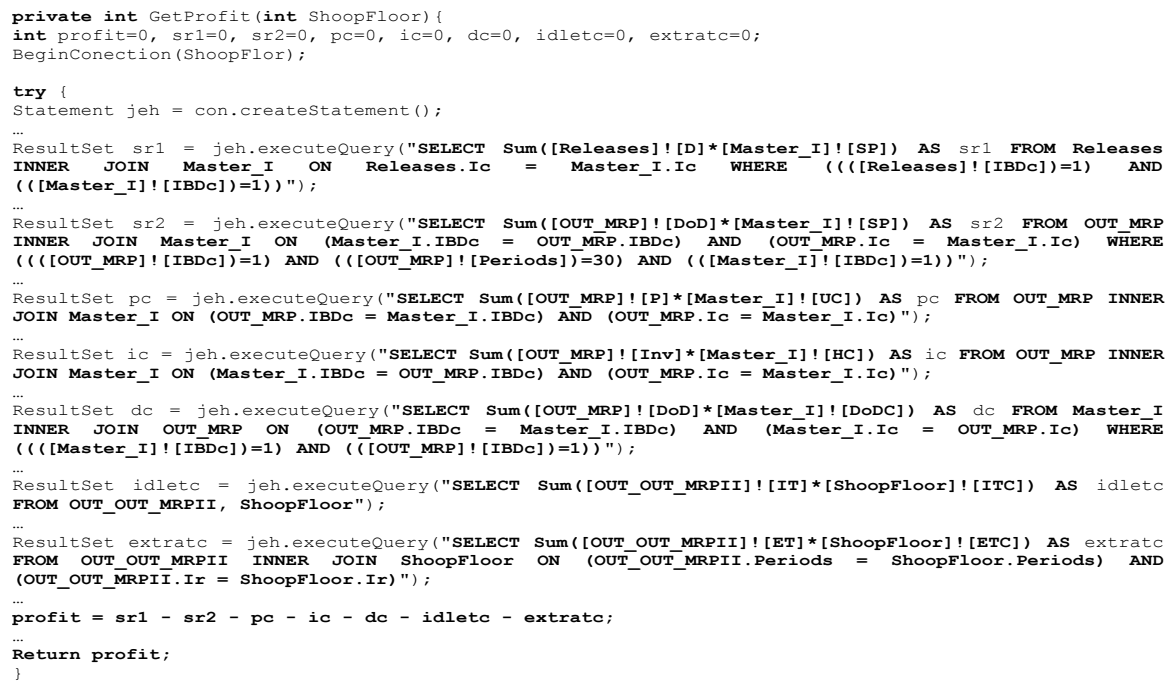

Fig. 4. JAVA - Extract of the SQL profit calculus code

Then, the negotiation process supported by the profit algorithm calculus (8) is presented in its technical JAVA-SQL approach (see Fig. 4). Fig. 5 presents the communication process which considers the main planning decision relating to the negotiation process (supported by the profit level). 
Therefore from a decentralized perspective, the system implementation, as Fig. 5 highlights, considers that each agent in the collaborative planning supported by the multi-agent system is in its own machine; that is, in its own container. Thus, each thread is composed of sub-threads that are supported by the relating FIPA contract-net protocols. Every agent belonging to the highest level will wait for the negotiated answers from the lower levels, and each node will evaluate its profit evolution.

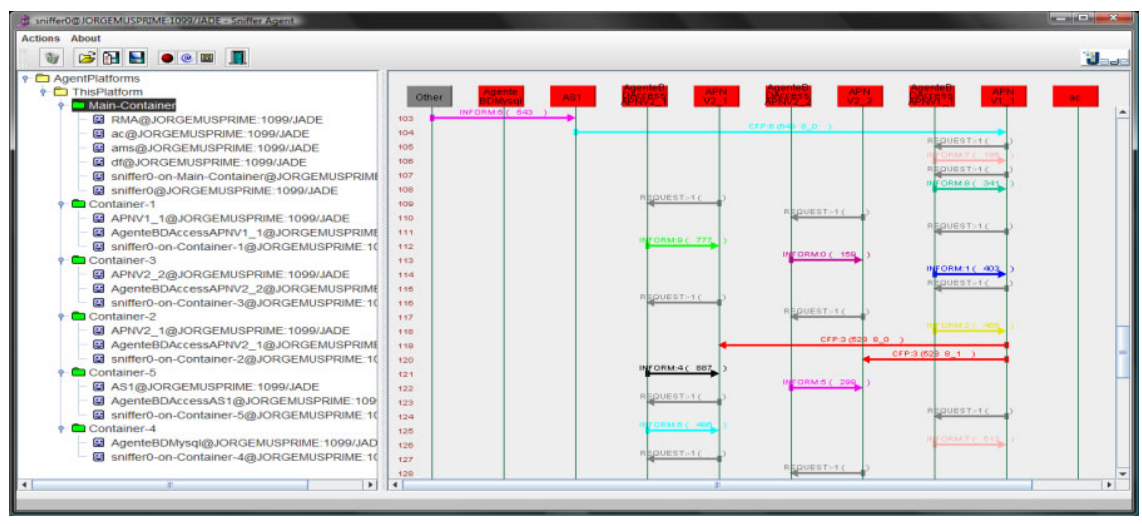

Fig. 5. Sniffer agent. Validation of the collaborative planning process

\section{Conclusions}

A novel multiagent-based collaborative planning model for multi-level supply chains has been proposed. The modeling basis is supported by the fact that each node considers its own information repository from a decentralized viewpoint, hence its own databases. In addition, the MRP mechanism supports the decision-making processes. The main idea is that these mechanisms will consider some inputs from the lower levels of the supply chain (suppliers) to, in turn, consider their answers about their profit calculus. Hence, the negotiation process will be composed of both the inputs and outputs relating to the decision, and the answers from all the supply chain levels; and all this from a decentralized perspective. In addition, a prototype has been developed that offers a good possibility of establishing the multi-agent system.

In future research, the proposed model will be applied to a real supply chain network in the automobile supply chain sector with a view to implementing the mobility agent by scaling the platform prototype and by considering real demand data. Furthermore, this novel proposal will also be compared with other negotiation mechanisms to test its suitability.

Acknowledgments. This research has been supported by the EVOLUTION project (Ref. DPI2007-65501) which is funded by the Spanish Ministry of Science and Education, www.cigip.upv.es/evolution. 


\section{References}

1. Chaharsooghi, S.K., Heydari, J.: LT variance or LT mean reduction in supply chain management: Which one has a higher impact on SC performance. International Journal of Production Economics 124, 475-481 (2010)

2. Dudek, G., Stadtler, H.W.: Negotiation-based collaborative planning between supply chains partners. European Journal of Operational Research 163(3), 668-687 (2005)

3. Gaudreault, J., Frayret, J., Pesant, G.: Distributed search for supply chain coordination. Computers in Industry 60, 441-451 (2009)

4. Gomez-Gasquet, P., Franco, R.D., Rodríguez, R., Ortiz, A.: A Scheduler for extended supply chains based on combinatorial auctions. Journal of Operations and Logistics 12 , V1-V12 (2009)

5. Gupta, A., Maranas, C.D.: Managing demand uncertainty in supply chain planning. Computers and Chemical Engineering 27, 1219-1227 (2003)

6. Hernández, J.E., Mula, J., Ferriols, F.J.: A reference model for conceptual modeling of production planning processes. Production Planning \& Control 19(8), 725-734 (2008)

7. Hernández, J.E., Alemany, M.M.E., Lario, F.C., Poler, R.: SCAMM-CPA: A Supply Chain Agent-Based Modelling Methodology That Supports a Collaborative Planning Process. Innovar. 19(34), 99-120 (2009)

8. Hernández, J.E., Poler, R., Mula, J., Lario, F.C.: The reverse logistic process of an automobile supply chain network supported by a collaborative decision-making model. Group Decision and Negotiation Journal (2010) (in Press)

9. Jung, H., Chen, F.F., Jeong, B.: Decentralized supply chain planning framework for third party logistics partnership. Computers \& Industrial Engineering 55, 348-364 (2008)

10. Nahm, Y.E., Ishikawa, H.: A hybrid multi-agent system architecture for enterprise integration using computer networks. Robotics and Computer-Integrated Manufacturing 21, 217-234 (2005)

11. Ortiz, A., Franco, R.D., Alba, M.: V-Chain: Migrating From Extended To Virtual Enterprise Within An Automotive Supply Chain. In: PRO-VE 2003, Proceedings. Processes and Foundations for Virtual Organizations, pp. 145-152 (2003)

12. Ouhimmou, M., D’Amours, S., Beauregard, R., Ait-Kadi, D., Chauhan, S.S.: Furniture supply chain tactical planning optimization using a time decomposition approach. European Journal of Operational Research 189, 952-970 (2008)

13. Poler, R., Hernández, J.E., Mula, J., Lario, F.C.: Collaborative forecasting in networked manufacturing enterprises. Journal of Manufacturing Technology Management 19(4), 514-528 (2008)

14. Sadeh, N.M., Hildum, D.W., Kjenstad, D., Tseng, A.: MASCOT: An Agent-Based Architecture for Coordinated Mixed-Initiative Supply Chain Planning and Scheduling. In: Third International Conference on Autonomous Agents. Workshop on Agent-Based Decision Support for Managing the Internet-Enabled Supply Chain, Seattle WA (1999)

15. Su, Y., Yang, C.: Why are enterprise resource planning systems indispensable to supply chain management? European Journal of Operational Research 203, 81-94 (2010)

16. Xiao, T., Shi, K., Yang, D.: Coordination of a supply chain with consumer return under demand uncertainty. International Journal of Production Economics 124, 171-180 (2010)

17. Zhao, X., Lee, T.: Developments and emerging research opportunities in operations strategy and supply chain management. International Journal of Production Economics 120, $1-4(2009)$ 